\title{
Machine Learning for Geriatric Clinical Care: Opportunities and Challenges
}

\author{
Nazila Javadi-Pashaki ${ }^{1,2}$, Mohammad Javad Ghazanfari ${ }^{3}$, Samad Karkhah ${ }^{4}$ \\ ${ }^{1}$ Social Determinants of Health Research Center (SDHRC), Guilan University of Medical Sciences, Rasht, Iran \\ ${ }^{2}$ Department of Nursing, Cardiovascular Diseases Research Center, School of Nursing and Midwifery, Guilan University of Medical Sciences, Rasht, Iran \\ ${ }^{3}$ Department of Medical-Surgical Nursing, School of Nursing and Midwifery, Kashan University of Medical Sciences, Kashan, Iran \\ ${ }^{4}$ Department of Medical-Surgical Nursing, School of Nursing and Midwifery, Guilan University of Medical Sciences, Rasht, Iran
}

\section{To the Editor,}

Increasing life expectancy and geriatric-related changes pose major challenges for healthcare. ${ }^{1)}$ Geriatric patients experience many disorders including chronic diseases, weakness, cognitive decline, and functional dependence in the last two decades of life. As these problems can lead to hospitalization, these patients require quality clinical care. ${ }^{2)}$ Therefore, effective strategies for improving geriatric clinical care are essential. ${ }^{1)}$

One strategy to improve patient and clinical team outcomes, reduce costs, and enhance the health of patients is the use of artificial intelligence (AI). ${ }^{3)} \mathrm{AI}$ can play key roles in the prevention, diagnosis, and treatment of patients' problems and the provision of healthcare. ${ }^{4)} \mathrm{AI}$ has been used to detect cancer, disease management using robot-related technologies, provide screening tools to identify the risks of falls and urinary tract infections in geriatric patients with dementia, and assist healthcare providers in clinical decision-making and patient monitoring. ${ }^{5)}$ AI fills the gap of human resources in geriatric clinical care, reducing the burden on their family caregivers and, ultimately, improving the quality of care and life of older adults. ${ }^{6}$ Machine learning (ML) is one of the main components of AI that uses statistical techniques to allow computer programs to make decisions and predictions based on previous data and experiences. ${ }^{6}$ Recently, special attention has been paid to ML in the medical literature. Thus far, ML has been used to identify older people at high risk for dementia; predict weakness, risk of falls, pneumonia, delirium, and acute kidney injury; and provide geriatric clinical care to prevent these problems. ${ }^{7)}$ Moreover, these well-developed models show accuracy surpassing that of humans. ${ }^{8)}$

Many opportunities exist for the implementation of ML to improve geriatric care in the clinical setting. These opportunities include clinical task automation, optimizing clinical decision-making and support in practice, expanding clinical capacity, improving the safety level of geriatric patients, and increasing the quality of their care; ${ }^{9)}$ telemedicine and robot-related technologies for social communication and rehabilitation of geriatric patients with physical disabilities, Parkinson disease, and chronic hemiparetic gait as well as for post-stroke patients; virtual reality environments to improve anxiety, depression, loneliness, social isolation, gait, posture, and pain; ${ }^{7)}$ and interpretation of motion parameters and assessment of body composition or physical performance from vision datasets or opportunistic imaging datasets. ${ }^{9,10)}$ Therefore, health managers and policymakers should pay special attention to ML to improve geriatric clinical care. ${ }^{11)}$

However, the implementation of ML for geriatric clinical care has challenges including causality, missingness, and outcome definition. Data collection is often performed in a clinical setting via observation. The application of observational data to answer causal questions in a clinical setting is challenging. However, observational data may provide information that is often ignored. Therefore, learning models that do not consider missingness indicators can lead to inaccurate assessments and reflections of human biases. In contrast, reliable learning outcomes play an important role in assigning tasks. The key factors in outcome definitions in clinical settings are reliable outcomes, perception of clinical relevance, and the subtlety of label leakage, which can be effective in the implementation of ML for geriatric clinical care. ${ }^{11)}$ Some additional challenges of using ML for geriatric clinical care include ethical concerns such as racial biases, ${ }^{12)}$ privacy, equity, security, disruption of human communication and data management, and cost of care. ${ }^{7)}$ One of the major challenges in the implementation of $M L$ for geriatric clinical care is the problem of annotation of real-life emotions. Data related to the expression of emotions are rare and cannot provide a basis for annotation and modeling using fine-grained emotion labels. However, inter-labeler agreement and annotation label confidence are important factors that need to be addressed to solve this challenge. ${ }^{13)}$ Therefore, these challenges remain a major con- 
cern for the implementation of ML in geriatric clinical care. ${ }^{7)}$

Overall, the implementation of $\mathrm{ML}$ to improve geriatric clinical care is a questionable hypothesis requiring additional evidence. Thus, further research is needed to address the challenges of using $\mathrm{ML}$ for geriatric clinical care.

\section{ACKNOWLEDGMENTS}

\section{CONFLICT OF INTEREST}

The authors claim no conflicts of interest.

\section{FUNDING}

None.

\section{AUTHOR CONTRIBUTION}

Conceptualization, SK, NJP; Data curation, SK, NJP, MJG; Investigation, SK, NJP, MJG; Methodology, SK, NJP; Project administration, SK; Supervision, NJP; Writing-original draft, NJP, MJG, SK; Writing-review \& editing, SK, NJP.

\section{REFERENCES}

1. Javadi-Pashaki N, Mohammadi F, Jafaraghaee F, Mehrdad N. Keeping up with the caravan of life: successful aging strategies for Iranian women. Int J Qual Stud Health Well-being 2015; $10: 29500$

2. Voumard R, Rubli Truchard E, Benaroyo L, Borasio GD, Bula C, Jox RJ. Geriatric palliative care: a view of its concept, challenges and strategies. BMC Geriatr 2018;18:220.

3. Matheny ME, Whicher D, Thadaney Israni S. Artificial intelligence in health care: a report from the National Academy of Medicine. JAMA 2020;323:509-10.

4. Emanuel EJ, Wachter RM. Artificial intelligence in health care: will the value match the hype? JAMA 2019;321:2281-2.

5. Choudhury A, Renjilian E, Asan O. Use of machine learning in geriatric clinical care for chronic diseases: a systematic literature review. JAMIA Open 2020;3:459-71.

6. Ho A. Are we ready for artificial intelligence health monitoring in elder care? BMC Geriatr 2020;20:358.

7. Makaetova-Ladinska E, Harwood T, Maltby J. Artificial Intelligence in the healthcare of older people. Arch Psychiatry Ment Health 2020;4:7-13.

8. Sendak M, Gao M, Nichols M, Lin A, Balu S. Machine learning in health care: a critical appraisal of challenges and opportunities. EGEMS (Wash DC) 2019;7:1.

9. Skeppstedt M, Kvist M, Nilsson GH, Dalianis H. Automatic recognition of disorders, findings, pharmaceuticals and body structures from clinical text: an annotation and machine learning study. J Biomed Inform 2014;49:148-58.

10. Prakash C, Kumar R, Mittal N. Recent developments in human gait research: parameters, approaches, applications, machine learning techniques, datasets and challenges. Artif Intell Rev 2018;49:1-40.

11. Ghassemi M, Naumann T, Schulam P, Beam AL, Chen IY, Ranganath R. A review of challenges and opportunities in machine learning for health. AMIA Jt Summits Transl Sci Proc 2020; 2020:191-200.

12. Char DS, Shah NH, Magnus D. Implementing machine learning in health care: addressing ethical challenges. N Engl J Med 2018;378:981-3.

13. Devillers L, Vidrascu L, Lamel L. Challenges in real-life emotion annotation and machine learning based detection. Neural Netw 2005; 18:407-22.

Corresponding Author: Samad Karkhah, MSc

Department of Medical-Surgical Nursing, School of Nursing and Midwifery, Guilan University of Medical Sciences, Rasht, Iran

E-mail: sami.karkhah@yahoo.com

ORCID: https://orcid.org/0000-0001-9193-9176

Received: May 29, 2021; Revised:June 5, 2021; Accepted:June 5, 2021 